\title{
Tibial stress injuries - location, severity, and classification in magnetic resonance imaging examination
}

\author{
Agata Maria Gmachowska ${ }^{1,2 A, B, C, C, E, F}$, Magdalena Żabicka ${ }^{2,3 A, D, E, F}$, Ryszard Pacho ${ }^{2,4 A}$, Szymon Pacho ${ }^{5 E}$, \\ Aleksandra Majek ${ }^{1 \mathrm{~F}}$, Beata Feldman ${ }^{1,2 \mathrm{~F}}$ \\ 'Department of Radiology, Gruca Orthopaedic and Trauma Teaching Hospital, Centre of Postgraduate Education, Otwock, Poland \\ ¿Luxmed Diagnostyka, Warsaw, Poland \\ ${ }^{3}$ Department of Radiology, Military Institute of Medicine, Warsaw, Poland \\ ${ }^{4}$ II Department of Clinical Radiology, Medical University of Warsaw, Warsaw, Poland \\ ${ }^{5}$ Department of Internal Medicine \& Cardiology, Medical University of Warsaw, Warsaw, Poland
}

\section{Abstract}

Purpose: To describe and illustrate the spectrum of magnetic resonance imaging (MRI) findings of tibial stress injuries (TSI) and propose a simplified classification system.

Material and methods: Retrospective analysis of MRI exams of 44 patients with clinical suspicion of unilateral or bilateral TSI, using a modified classification system to evaluate the intensity and location of soft-tissue changes and bone changes.

Results: Most of the patients were young athletic men diagnosed in late stage of TSI. Changes were predominantly found in the middle and distal parts of tibias along medial and posterior borders.

Conclusions: TSI may be suspected in young, healthy patients with exertional lower leg pain. MRI is the only diagnostic method to visualise early oedematic signs of TSI. Knowledge of typical locations of TSI can be helpful in proper diagnosis before its evolution to stress fracture.

Key words: stress, fractures, soft tissue injuries, athletic injuries, tibial stress syndrome, tibial fractures.

\section{Introduction}

Stress or fatigue injuries are a growing problem in sports medicine because of the rise in popularity of sport activities and active lifestyle. These injuries are defined as a reaction of normal bone to abnormal repetitive stress [1] and should be distinguished from insufficiency fractures, which result from normal stress to abnormal bone (for example in elderly patients with osteoporosis, patients with anorexia, and others). Many bones may be affected by stress injuries, depending on the type of sport activity and acting forces. Stress injuries are frequent in runners and military recruits but may also be associated with other sports and activities (swimming, skating, aerobics, ballet dancing, etc.). Tibial stress injury (TSI) is responsible for up to $75 \%$ of exertional leg pain [1]. Among the 10 most common types of injury in runners TSI is the only type of injury affecting the bone [2]. Very often TSI is related to training errors, such as sudden increase in training volume. Change of training surface or inappropriate shoes may also result in TSI [1-3].

TSI may be suspected in young, healthy runners with exertional lower leg pain. Patients with TSI present unilateral or bilateral lower leg pain, relieved by rest. The most

\section{Correspondence address:}

Agata Maria Gmachowska, Department of Radiology, Gruca Orthopaedic and Trauma Teaching Hospital, Centre of Postgraduate Education, Konarskiego 13,

05-400 0twock, Poland, e-mail: agmachowska@op.pl

Authors' contribution:

A Study design · B Data collection · C Statistical analysis · D Data interpretation · E Manuscript preparation · F Literature search · G Funds collection 
common signs of stress injuries are focal tenderness and soft tissue swelling.

Imaging options of TSI start with radiography and ultrasonography. In early stages of TSI they might be negative but may rule out other abnormalities (such as muscle or tendon injuries, neoplasms, and others). MRI is the only diagnostic method to visualise early oedematous signs of TSI $[1,3,4]$ - even subtle periosteal and bone marrow oedema. Severity of changes in magnetic resonance imaging (MRI) corresponds with clinical severity [5]. MRI findings must be compared with signs and symptoms present during clinical examination, medical history of the patient, laboratory investigation, and other exams (ultrasonography, radiological examination, computed tomography - CT, bone scintigraphy). In non-specific cases (i.e. suspicion of neoplasm or infection), contrast administration may be required. Both marrow and periosteal oedema are enhanced after contrast administration and in some cases may be difficult to distinguish from neoplastic changes. No evidence of soft tissue mass or pathological destruction of the bone and knowledge of typical location of TSI can be helpful in making the correct diagnosis. MRI examination is the only method to identify early symptoms of stress injuries (periosteal and bone marrow oedema), even when a fracture line is not visible. CT may be helpful in visualising changes in cortex in doubtful cases.

In 1995 Fredericson et al. [3] proposed the five-point grading system of MRI findings of TSI. Kijowski et al. [4] performed a study for validation of the Fredericson MRI classification of TSI, comparing MRI features of injury severity with clinical outcome. They used the modified Fredericson classification of TSI composed of six grades, dividing grade 4 into grade $4 \mathrm{a}$ with cortical abnormalities and $4 \mathrm{~b}$ where the fracture line is visible. The results of their work showed that in grades 2, 3, and 4a the severity of periosteal and bone oedematous changes and clinical prognosis where similar.

The purpose of our study was to describe and illustrate the location and spectrum of MRI findings of TSI in healthy active people and to classify the visible MRI changes. We propose a simplification of the grading system, based on the classifications made by Frederickson, Gaetta, and Kijowski, with modifications made by Beck et al. [5].

Table 1. Five-points grading system of TSI

\begin{tabular}{|l|l|}
\hline Grade of stress injury & MRI findings \\
\hline 0 & No abnormality \\
\hline 1 & Presence of periosteal oedema only \\
\hline 2 & Periosteal and bone marrow oedema \\
\hline 3 & Grade 2 and cortical abnormalities \\
\hline 4 & Grade 3 and fracture line \\
\hline
\end{tabular}

\section{Material and methods}

Forty-four patients with clinical suspicion of TSI underwent MRI examination. The study group consisted of 14 women and 30 men, aged from 13 to 61 years (mean age 35.3 years). In total 53 tibiae (one tibia in 35 patients and two tibiae in nine patients) were examined. Most of the patients were recreational runners, one of them was a triathlete, two were athletes, and one was a professional skier. Their symptoms lasted from weeks to months. The type and location of changes were analysed independently by two radiologist with eight and 15 years of experience in musculoskeletal radiology, and a common opinion on the severity of changes was established afterwards.

The examinations were performed on three $1.5 \mathrm{~T}$ scanners: Signa (General Electric), Symphony mobile (SIEMENS), and Avanto (SIEMENS). One or both lower legs were scanned with the subject in a supine position from knee to talar joint using a flex body coil. The scanning protocols included: T1-WI (spin-echo; TR $=500$ $670 \mathrm{~ms}, \mathrm{TE}=9.4-10.2 \mathrm{~ms}$ ) in axial, sagittal, and coronal plane, PD-WI fat saturated (spin-echo TR $=2300-3360$ $\mathrm{ms}, \mathrm{TE}=37.0-46.9 \mathrm{~ms}$ ) in axial and coronal plane, $\mathrm{T} 2$ $\mathrm{WI}$ (spin-echo TR $=3550-4800 \mathrm{~ms}, \mathrm{TE}=89.5-99.0 \mathrm{~ms}$ ) in sagittal plane, fat saturated inversion recovery images (T2 STIR TR $=4800 \mathrm{~ms}$, TE $=40.7 \mathrm{~ms}$, TI $=150 \mathrm{~ms}$ ), or fat saturated T2 WI (T2 SPAIR TR $=4200 \mathrm{~ms}, \mathrm{TE}=97$ $\mathrm{ms}$ ) in coronal plane. The slice thickness, matrix, and field of view depended on the plane of examination. In axial plane, slice thickness $5 \mathrm{~mm}$ with a 7 -mm gap, matrix 512 $\times 304$ or $512 \times 512$ depending on the scanner and field of view adjusted to the space of examination $(20-35 \mathrm{~cm})$; in sagittal plane, slice thickness $3 \mathrm{~mm}$ with a 4-mm gap, matrix from $384 \times 384$ to $512 \times 512$ depending on the scanner and maximal possible field of view $(32-46 \mathrm{~cm})$; in coronal plane, slice thickness $3 \mathrm{~mm}$ with a $3.5 \mathrm{~mm}$ gap, matrix from $368 \times 512$ to $512 \times 512$ depending on the scanner and maximal possible field of view $(32-46 \mathrm{~cm})$.

To classify the types of changes a simple five-point grading system of TSI (Table 1) was used. It was based on classifications established by Fredericson et al. [3] and Kijowski et al. [4], supplemented by the conclusions concerning cortical abnormalities visible in MRI examination in patients with TSI made by Gaeta et al. [1] and modifications made by Beck et al. [5].

\section{Results}

There are four main MRI findings that may suggest stress injury. They can be divided into soft-tissue changes (periosteal oedema) and bone changes (bone marrow oedema, cortical abnormalities, and fracture line) $[1,3,4]$. We decided to use that as a basis of our classification.

Grade 1. The first MRI finding suggests TSI is periosteal oedema. It may be very subtle and noticeable in early stages, only on fluid-sensitive sequences (STIR, fat 

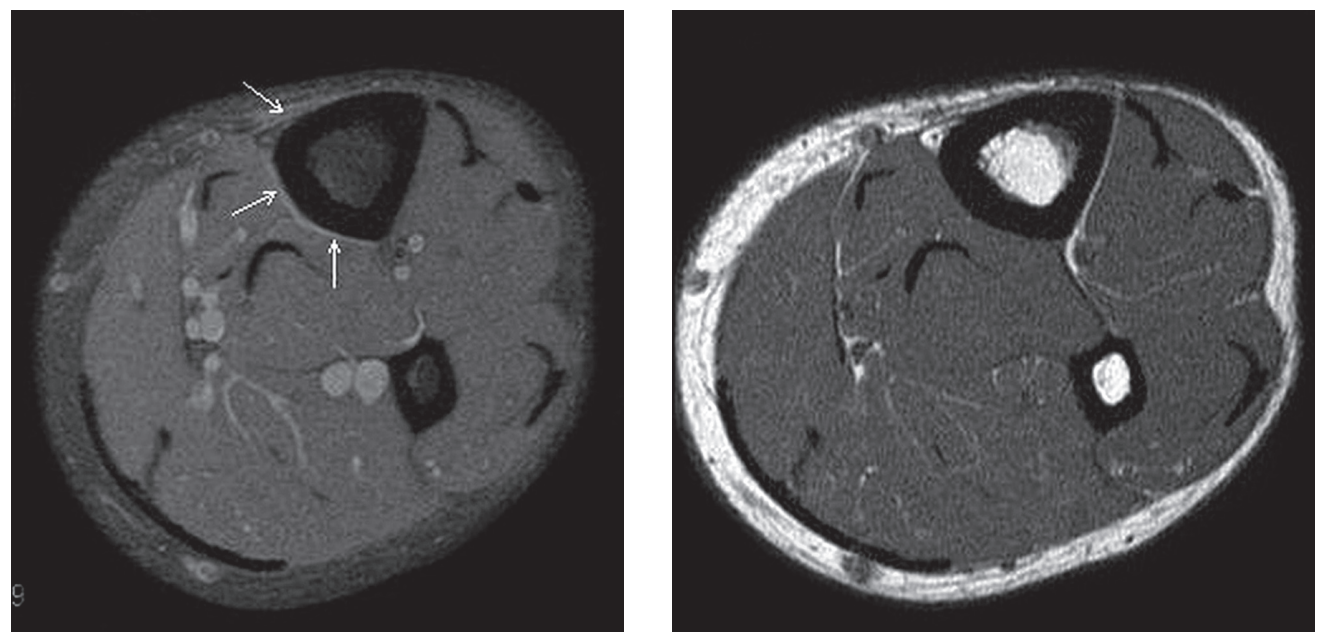

Figure 1. Grade 1 TSI: discrete periosteal oedema on medial and posterior side of distal part of left tibia, better seen on Ax PD fat sat images (arrows) than on Ax T1 FSE images. 46-year-old man with lower leg pain in last 10 days
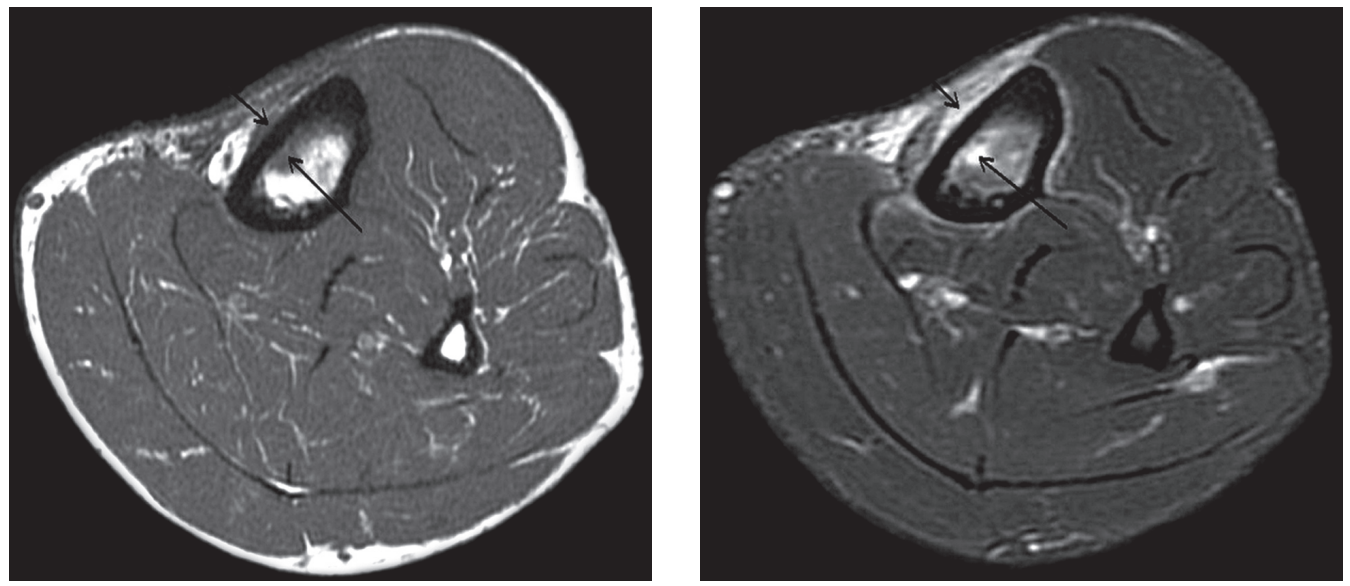

Figure 2. Grade 2 TSI: periosteal oedema and bone marrow oedema without cortical abnormalities (ax PD FatSat)

suppressed T2- and PD-WI) (Figure 1). When oedema extends, it is visible both on T1-WI and fluid-sensitive sequences. In our study we found periosteal oedema in eight cases.

Grade 2. In early stages bone marrow stays normal on all sequences. Chronic repetitive stress without proper rest causes stress reactions in bone, which begin with bone marrow oedema. It is usually accompanied by periosteal oedema (Figures 2,3) at similar level as periosteal oedema but usually on a shorter segment (Figure 3). Periosteal and bone marrow oedema was seen in five of our patients (grade 2).

Grade 3. Abnormal repetitive activity causes remodelling of the bone. It is caused by osteoclast-mediated resorption and osteoblastic replacement and leads to changes in cortex. Normal cortex has low signal intensity on T1-WI and T2-WI, and every change in intensity suggests remodelling of the bone. Gaeta et al. [1] described three types of cortical abnormalities visible in MRI and CT. Osteopaenia is defined as loss of cortical signal void (MRI)/ increased hypo attenuation (CT). The resorption cavity is a round or oval intracortical area of increased signal

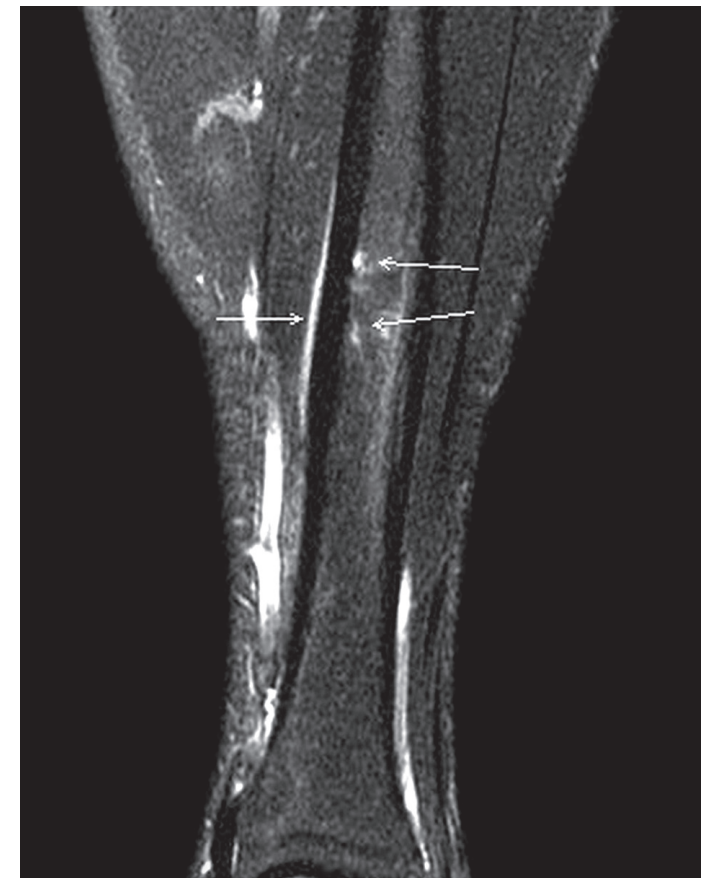

Figure 3. Another example of grade 2 TSI (Cor PD Fat Sat) - periosteal oedema and bone marrow oedema (arrows) 


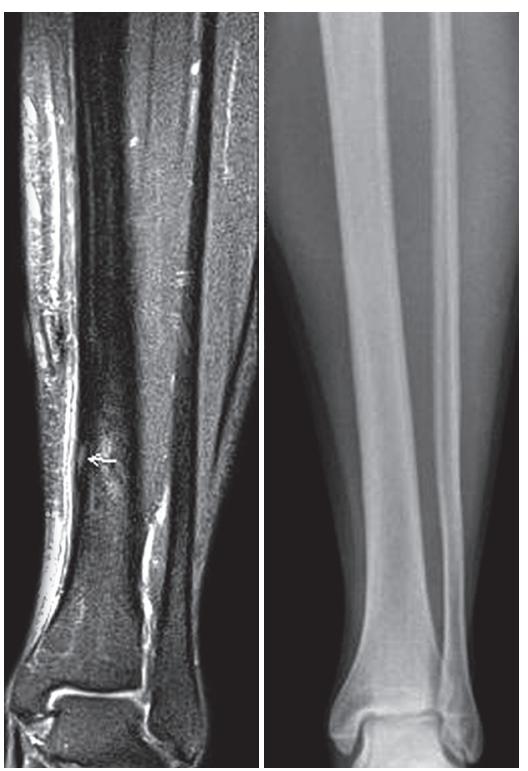

Figure 4. Grade 3 TSI: cortical abnormalities (arrow), periosteal and bone marrow oedema (Cor PD FatSat). Classical radiograph of the same patient on the right

intensity (MRI)/hypo attenuation (CT). Striation may be seen as subtle intracortical linear hyper intensity (MRI)/ hypo attenuation (CT). These abnormalities classified as grade 3 may be difficult to identify on MRI; usually they are better seen on CT scans. It is important to distinguish cortical abnormalities from small blood vessels crossing the cortex.
Cortical abnormalities (Figures 4-7) are usually accompanied by periosteal and bone marrow oedema, but oedematous changes are more extensive than changes in cortex (Figure 8). We found eight patients with grade 3 TSI.

Grade 4. Progressive and accelerated remodelling of the bone may lead to fracture. In some cases, the fracture line is difficult to visualise on T1 and T1 fat saturated images but can be revealed on PD fat saturated images and CT scans (Figure 9). The contrast medium administration can also help to visualise the fracture line in dubious cases (Figure 10). The fracture line is usually accompanied by periosteal, bone marrow, and sometimes muscle oedema. Finally, it spreads from the cortex to the marrow cavity. It may be better visualised in coronal and sagittal projections (Figures 11, 12) than on axial ones (Figure 13). It is essential to examine carefully all of the images and sequences to make the proper diagnosis. The majority of changes in our study (31) were classified as grade 4 .

TSI may be unilateral or bilateral. In bilateral TSI the changes are usually seen in different locations and stages (Figures 14, 15). Sometimes the severity of changes visible in MRI may be even more intense in the asymptomatic leg (Figure 16). Healing periosteal reactions begin after appropriate rest - then a subtle periosteal new bone formation (callus) may be detected (Figure 17). Usually the oedema and fracture line gradually disappear (Figure 18).

In conclusion, in our study 53 tibiae were examined; changes were seen in 52 locations (one tibia with changes

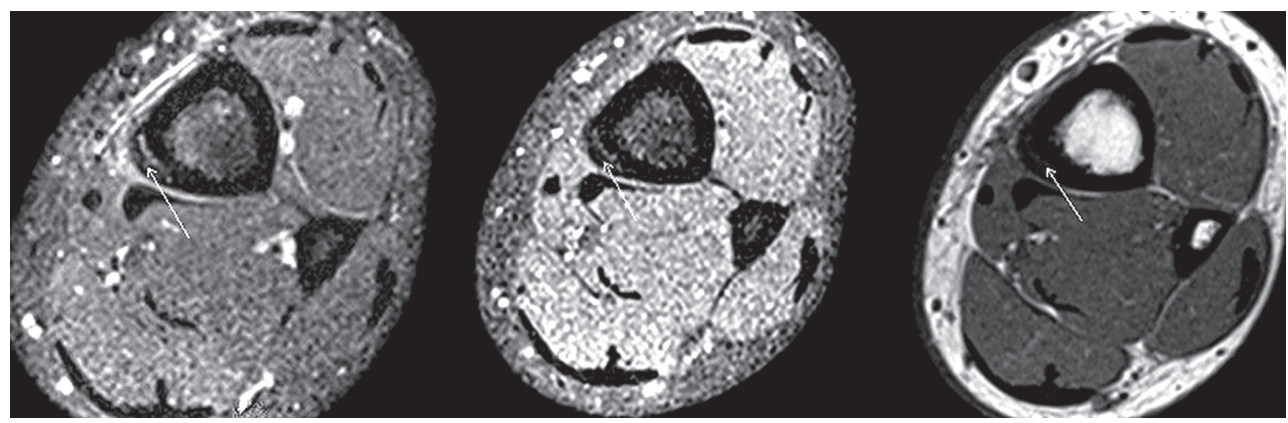

Figure 5. The same patient with grade 3 TSI-periosteal oedema with discrete bone marrow oedema. Arrows show thickened cortex with loss of cortical signal void (ax PD fatsat, T1 fatsat, T1)
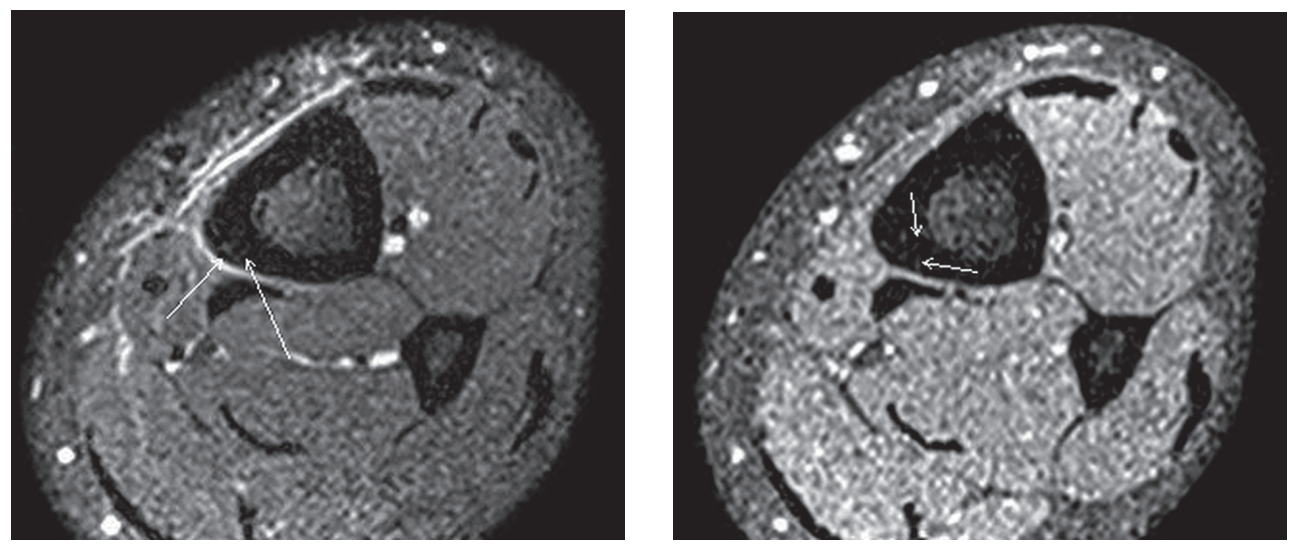

Figure 6. The same patient with grade $3 \mathrm{TSI}$ - arrows show subtle intracortical linear hyperintensity (striations) 

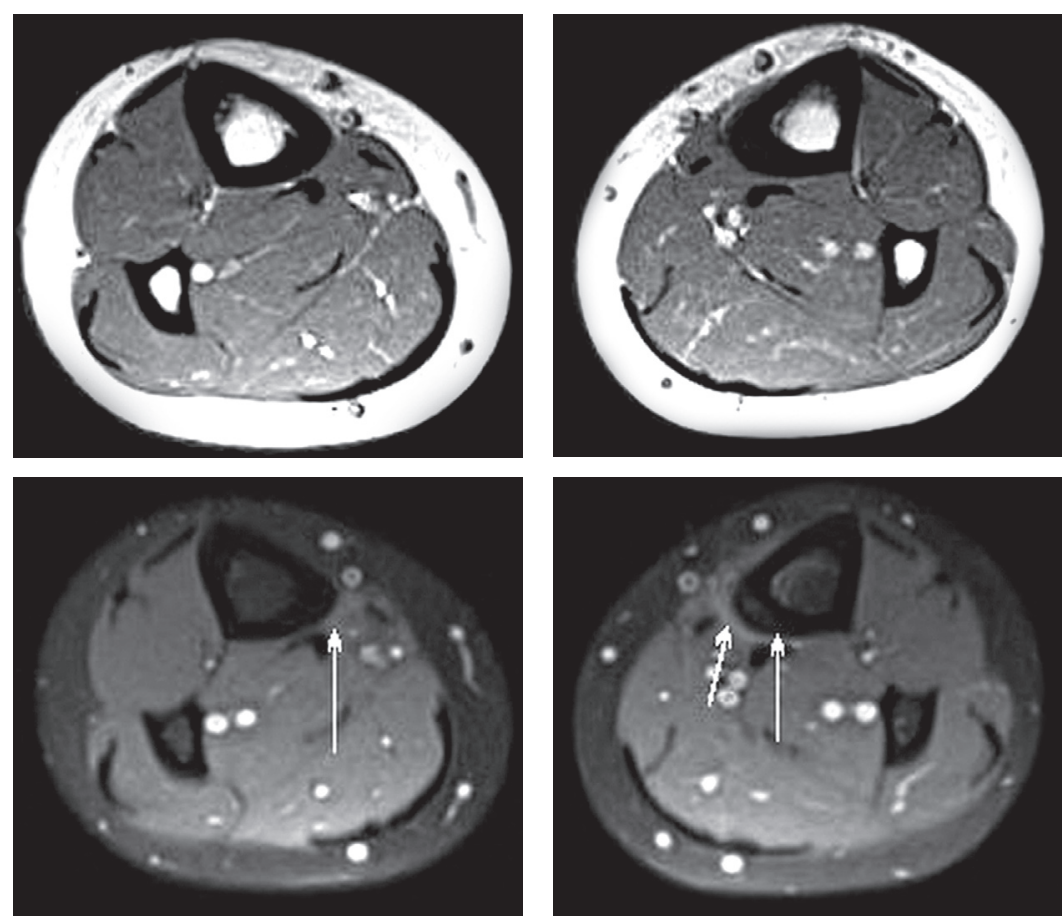

Figure 7. Bilateral TSI: grade 3 TSI on the left and grade 1 on the right. Left-sided periosteal and bone marrow oedema with cortical abnormalities on medio-posterior side. Subtle periosteal oedema on the right side in the same localisation

on two levels, and two of them showed no changes). In unilateral exams 19 right and 16 left tibiae were affected. In bilateral exams changes were seen in nine left tibias and right right ones (two tibiae with no abnormality).

The majority of the changes ( 31 among 52 locations) were classified as grade 4 (24 unilateral exams and seven bilateral exams). One patient was examined because of pain in right tibia - grade 1 TSI was found in the distal part of the tibia, but also grade 4 changes were shown in the proximal parts of both tibiae. Periosteal oedema was seen in eight cases (grade 1) - five in bilateral exams and three in unilateral exams. Periosteal and bone marrow oedema (grade 2) was seen in five cases - one in a bilateral exam and four in unilateral exams. Cortical abnormalities accompanied with oedematous changes (grade 3 ) were seen in eight cases (four unilateral and four bilateral exams). Only two cases of TSI grade 0 (no abnormality) were seen. The obtained results are shown in Table 2.

We analysed the location of TSI on longitudinal and axial plains.

On longitudinal plains we classified changes in three parts of the tibia: proximal, middle, and distal. Most of the abnormalities were found in middle and distal parts of the tibia (39 cases). In proximal parts only changes in grade 4 (14 cases) were found. Table 3 shows correlation between grade and location of the changes.

On axial planes MRI signs of TSI can be visible along the medial, posterior, and/or anterior border of the tibia. In our study most of the changes were seen in the medial and posterior parts of the tibia (27 cases - one case in grade 2 , seven in grade 3 , and 19 in grade 4 ). In 10 cases changes were localised on the anterior medial border (six

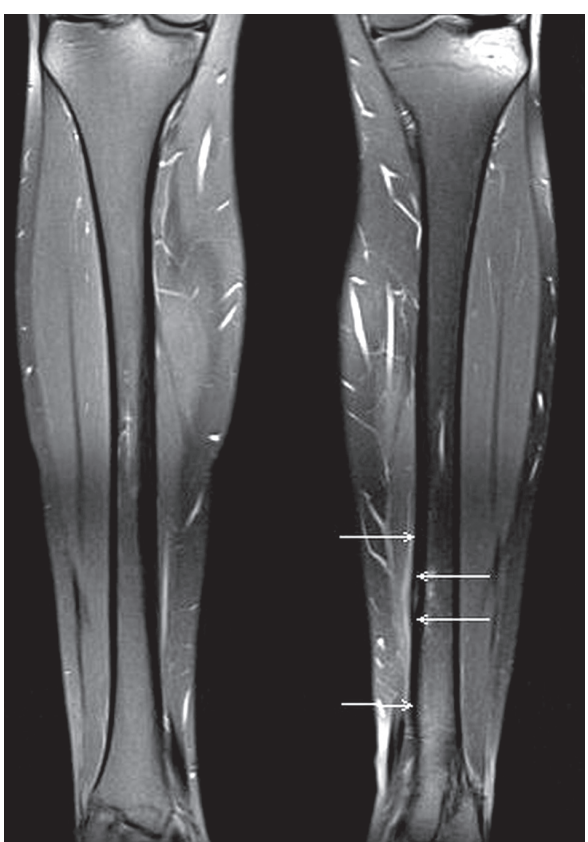

Figure 8. The same patient. Note that periosteal oedema is usually more extensive than changes in cortex

in grade 1 , one in grade 2 , one in grade 3 , and two in grade 4). In eight cases lesions were seen on the posterior border (seven in grade 4 and one in grade 3). In only four cases changes were seen on the anterior border (two cases in grade 1, two in grade 2) and three on the medial border (all in grade 4).

Additionally, in four cases with grade 4 lesions along the medial posterior border, there were also seen oedematous changes along the anterior border - at a similar level but on a longer span. Table 4 shows the correlation be- 

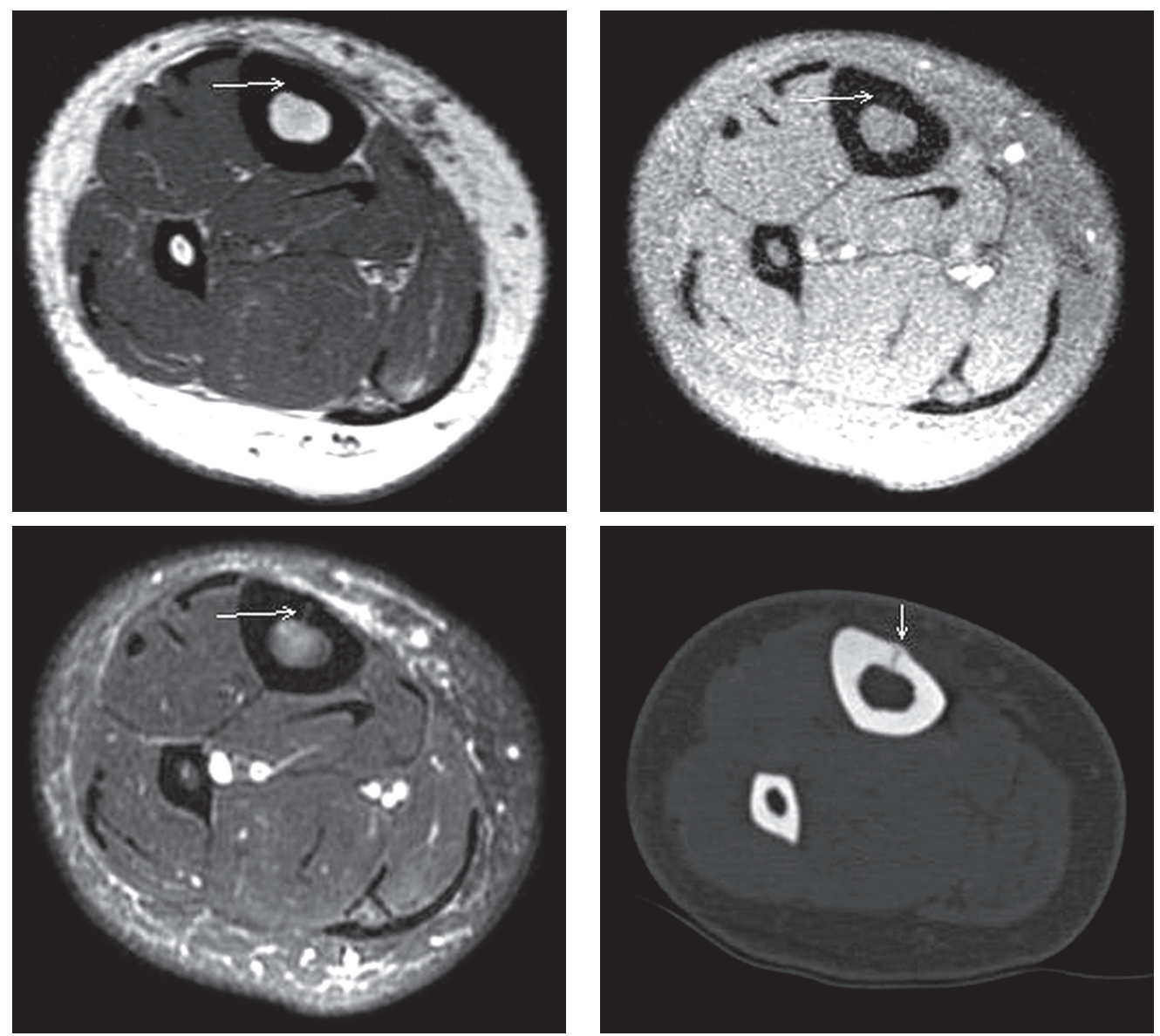

Figure 9. Grade 4 TSI. Fracture line can be difficult to visualise on T1 and T1 fat sat images (upper scans), but can be revealed on PD fat sat images and CT scans (lower scans). Changes in cortex are accompanied by periosteal oedema and subtle bone marrow oedema
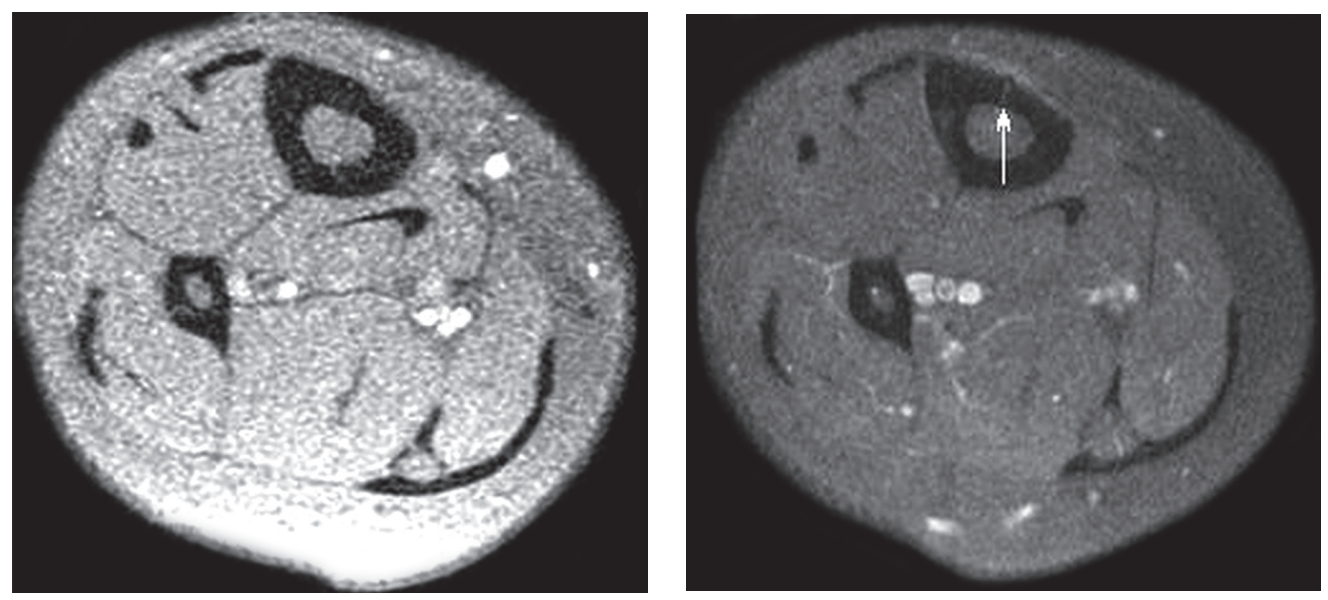

Figure 10. The same patient. Contrast medium administration can also help to visualise fracture line (ax T1 fat sat images without contrast on the left and with contrast on the right)

tween number of TSIs in each grade and location of the changes along the tibial border.

Most of our patients underwent an examination of one tibia (35). But in runners and other sportsmen both legs are usually under abnormal stress. In early stages only one tibia may be affected, but later the changes can be seen in both legs. Nine of our patients had two tibiae examined, and seven of them had bilateral changes seen on MRI. The severity (grade $0-4$ ) and location ( $\mathrm{P}$ - proximal, $\mathrm{M}$ - mid- dle, D - distal tibia) of changes seen in nine (1-9) patients with bilateral exams are shown in Table 5 .

\section{Discussion}

We proposed a simplified five-point grading system of TSI based on classifications established by Fredericson et al. [3] and Kijowski et al. [4], supplemented by the conclusions concerning the cortical abnormalities visible 

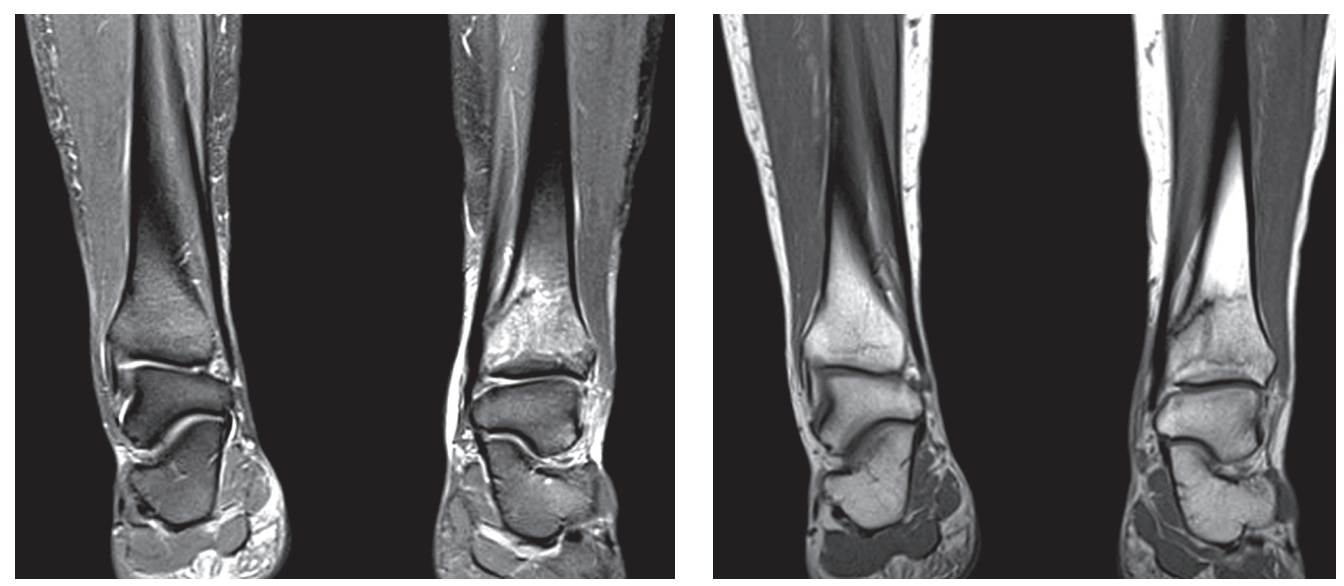

Figure 11. Grade 4 TSI. Fracture line on coronal images (PDfatsat, T1WI)

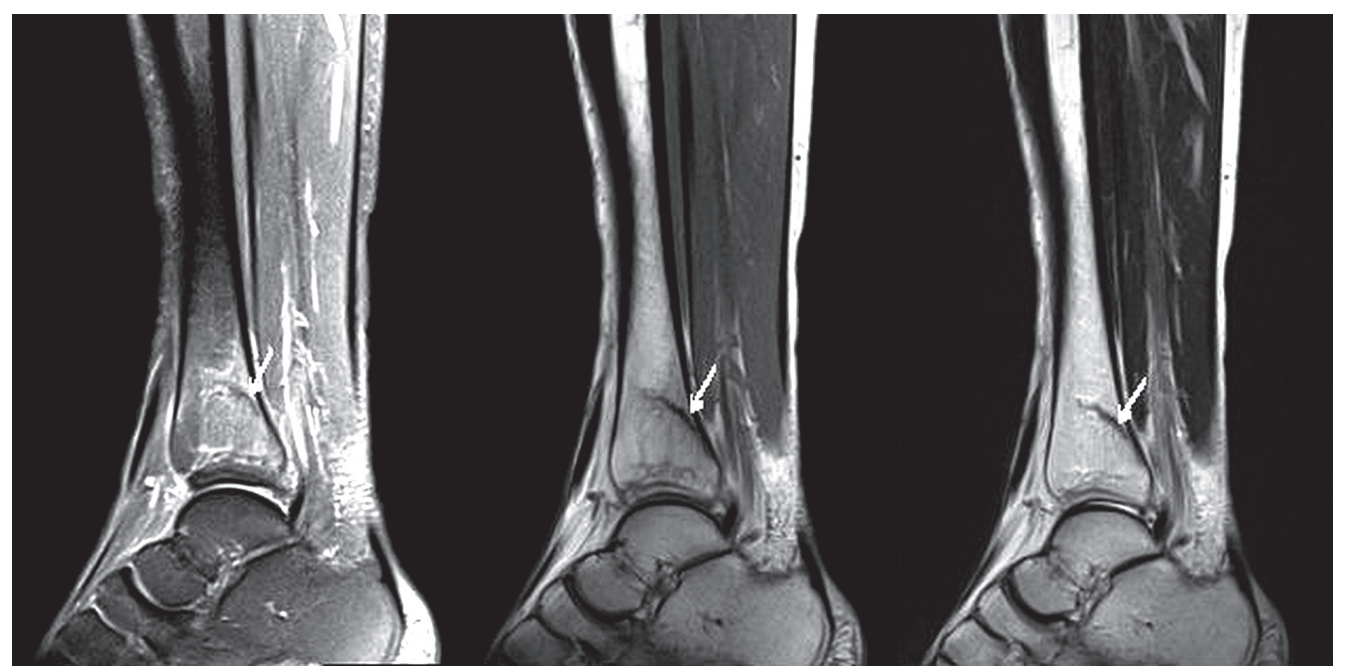

Figure 12. Grade 4 TSI. Fracture line on sagittal images (PDfatsat, T1WI)
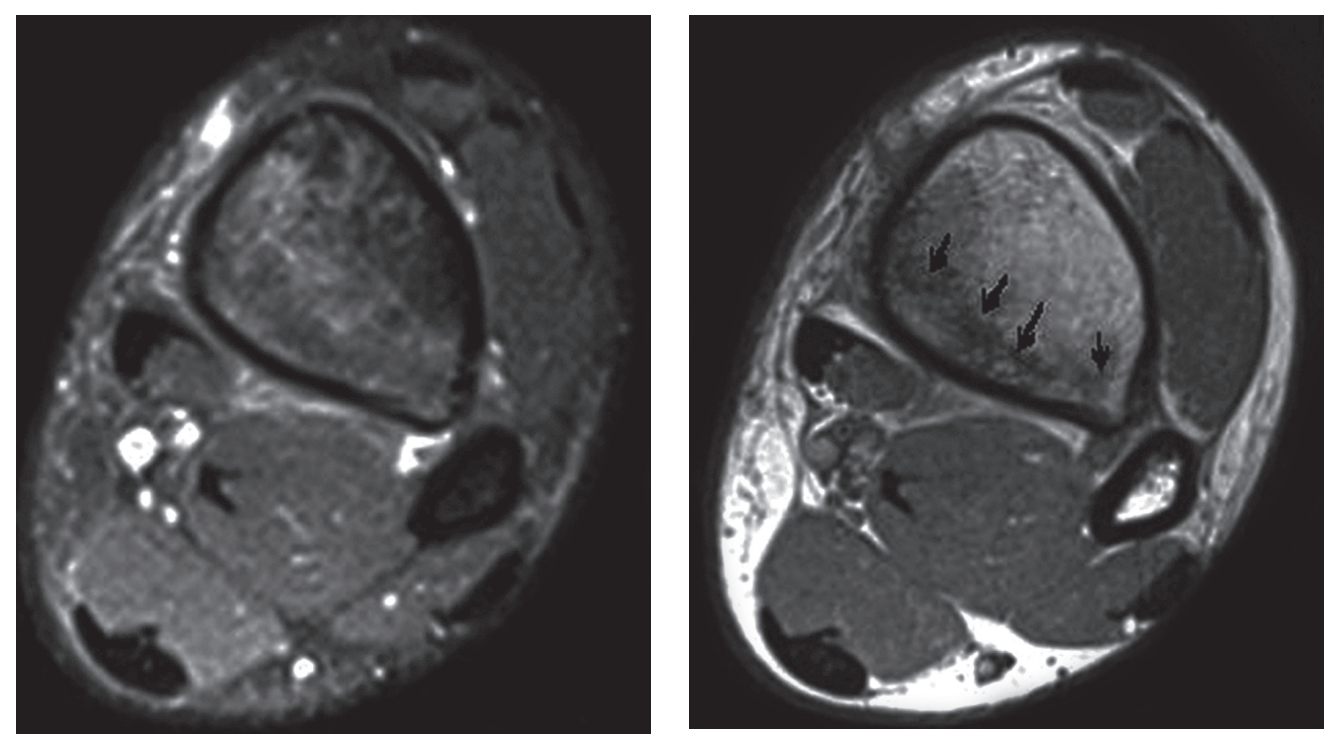

Figure 13. Grade 4 TSI. Fracture line on axial images (PDfatsat, T1WI)

in MRI examination in patients with TSI made by Gaeta et al. [1] and modified by Beck et al. [5].

Discussing the results of their work, Kijowski et al. [4] questioned the necessity to simplify the classification system of TSI and proposed the abbreviated three-grade Fredericson classification system for TSI: periosteal oedema only (grade 1), periosteal and bone marrow oedema (grade 2), and a liner intracortical fracture line (grade 3 ). 


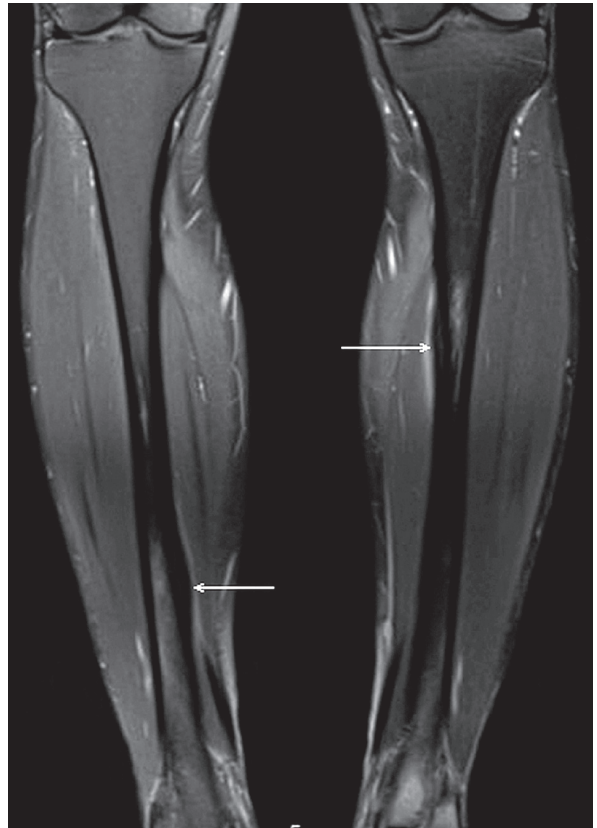

Figure 14. Bilateral TSI in 38-year-old runner. Cor PD fat sat image with proximal injury on the left and distal injury on the right (arrows)

Going along with their concern we decided to use the new but not so simple classification system of TSI, as they proposed. In our opinion, type 3 in the proposed classification of TSI corresponding to $4 \mathrm{a}$ in Fredericson classification should be separate because of different presentation in MRI examination. We did not analyse the time to re-
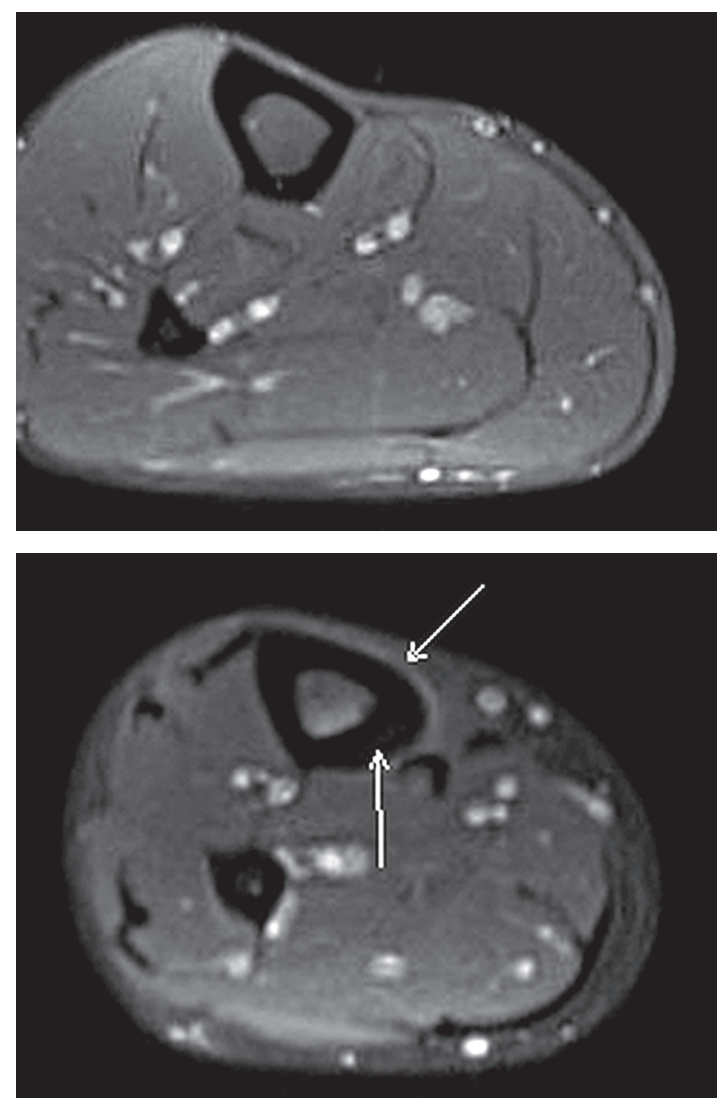

Figure 15. The same patient. Proximal left tibia with grade 4 TSI and fracture line (arrow) and distal right tibia with cortical abnormalities (grade 3). Note that periosteal and bone marrow oedema is more intense in the left tibia covery as the criterion of classification, so we agree with Kijowski et al. [4] that additional prospective studies are needed to determine the clinical utility of the proposed five-point or the abbreviated three-point Fredericson classification. In our grading system types 2 and 3 in the classification used by Kijowski et al. were fused in type 2; type 3 corresponds to type $4 \mathrm{a}$; and type 4 corresponds with type $4 b$.

The majority of the changes in our group (31 among 52 locations; $60 \%$ ) were classified as grade 4 . It may be caused by high motivation in training, without appropriate rest despite the pain. Oedematous changes on both sides of the cortex (grade 1 and 2) were less frequent, probably because patients with early stages of TSI chose to discontinue sport activity, which lead them to early recovery so they did not need any medical help.

Forty-five percent of cases in grade 4 (14 cases) were located in the proximal part (1/3) of the tibia, 39\% (12 cases) in distal tibia, and only $16 \%$ ( 5 cases) in middle tibia. The changes in grades $1-3$ in proximal tibia were not noticed; they were located in middle and distal tibia and were less frequent. The majority (84\%) of cases of TSI in our material were located in proximal and distal tibia. Kijowski et al. noticed that mid tibial diaphysis is most commonly involved in all grades of injury.

In the group of 55 asymptomatic recruits analysed by Hadid et al. [6] 26 had tibial stress changes in MRI examination in induction day and nine after a four-month
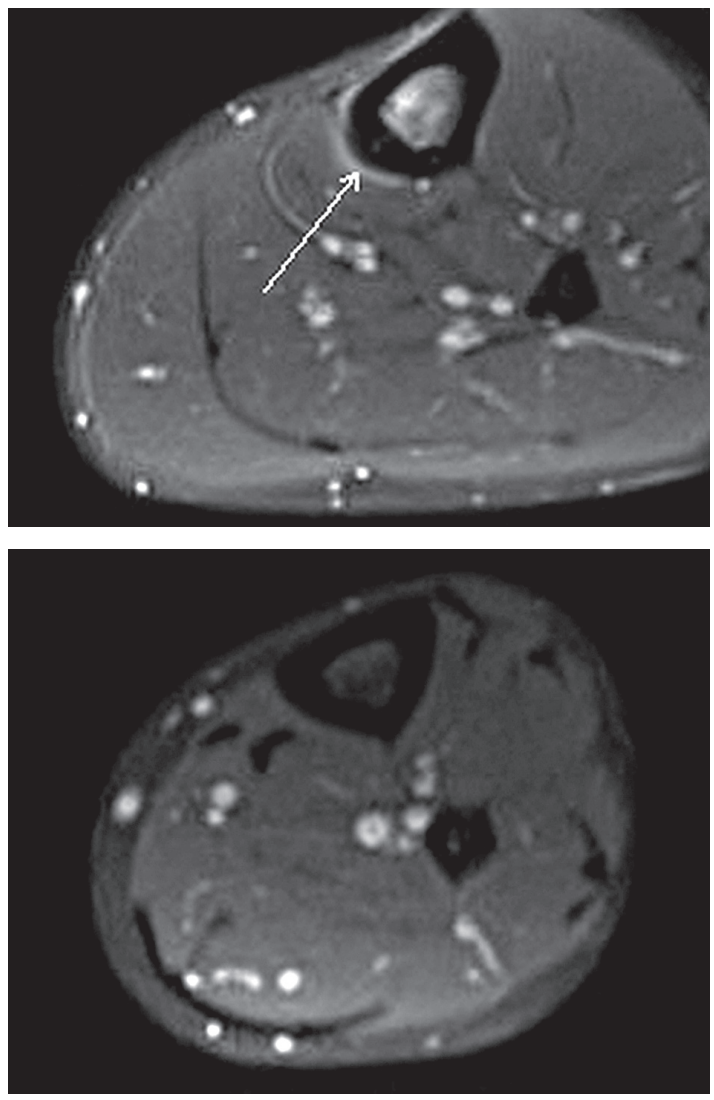


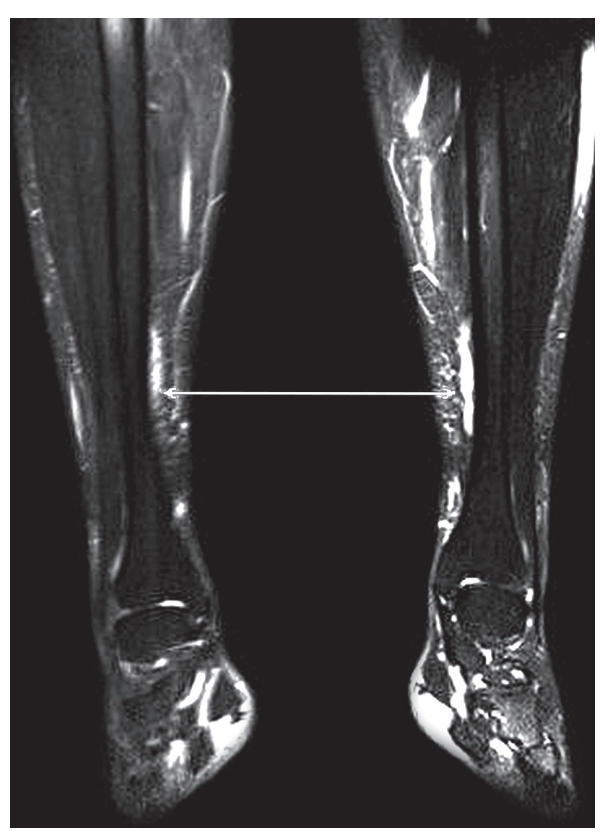

Figure 16. Bilateral periosteal oedema, more intense on the left (T2 SPAIR cor). No bone marrow oedema or cortical abnormalities were visible (grade 1 TSI). 33-year-old runner with right-sided lower leg pain

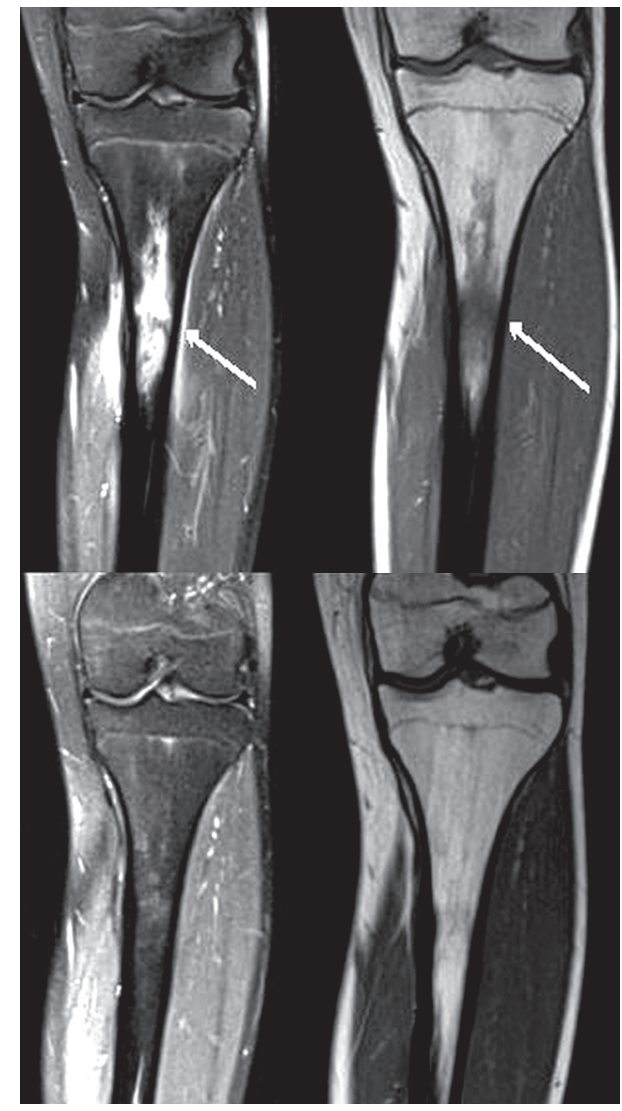

Figure 18. Upper images show patient after stress fracture, healed after 6 months of rest (lower images)

basic training, and most of them were endosteal marrow oedema (grade 2 in Fredericson grading system) located between the middle and distal thirds of the tibia. Before basic training the middle third of the tibia was the most frequent location of TSI (86\%), 14\% of changes were visible in proximal and none in the distal part of the tibia,

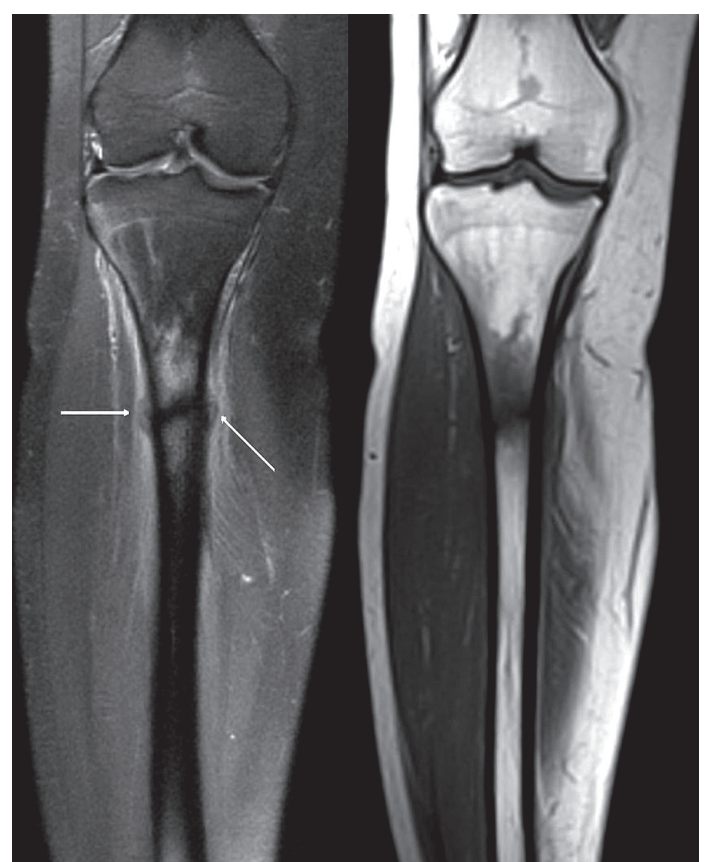

Figure 17. Healing periosteal reactions - a subtle periosteal new bone formation (callus)

Table 2. The obtained results of our study

\begin{tabular}{|l|c|}
\hline Grade & $\begin{array}{c}\text { Number of changes } \\
\text { in tibiae }\end{array}$ \\
\hline 0 - no abnormality & 2 \\
\hline 1 - presence of periosteal oedema only & 8 \\
\hline 2 - periosteal and bone marrow oedema & 5 \\
\hline 3 - grade 2 and cortical abnormalities & 8 \\
\hline 4 - grade 3 and fracture line & 31 \\
\hline Total & 52 \\
\hline
\end{tabular}

Table 3. Results: correlation between grade and location of TSI on longitudinal plains

\begin{tabular}{|l|c|c|c|c|}
\hline Grade & $\begin{array}{c}\text { Total number } \\
\text { of affected tibiae }\end{array}$ & $\begin{array}{c}\text { Proximal } \\
\text { tibia }\end{array}$ & $\begin{array}{c}\text { Middle } \\
\text { tibia }\end{array}$ & $\begin{array}{c}\text { Distal } \\
\text { tibia }\end{array}$ \\
\hline 1 & 8 & - & 3 & 5 \\
\hline 2 & 5 & - & 3 & 2 \\
\hline 3 & 8 & - & 2 & 6 \\
\hline 4 & 31 & 14 & 5 & 12 \\
\hline Total & 52 & 14 & 13 & 25 \\
\hline
\end{tabular}

and after a four-month basic training the majority (72\%) of TSI changes were localised in middle third of the tibia, $16 \%$ of cases in the proximal tibia and 13 changes in distal tibia. The results are different from those obtained in our work, and this fact is confusing for us, but we suspect this is due to the majority of more severe cases of TSI being in highly motivated runners observed in our material. Hadid et al. [6] concluded that most of tibial bone changes occurred before basic training smaller than $100 \mathrm{~mm}$ represent normal bone remodelling. 
Table 4. Results: location of TSI on axial plains

\begin{tabular}{|l|c|c|c|c|c|}
\hline Grade & $\begin{array}{c}\text { Anterior } \\
\text { border }\end{array}$ & $\begin{array}{c}\text { Anterior } \\
\text { and medial } \\
\text { border }\end{array}$ & $\begin{array}{c}\text { Medial } \\
\text { border }\end{array}$ & $\begin{array}{c}\text { Medial and } \\
\text { posterior } \\
\text { border }\end{array}$ & $\begin{array}{c}\text { Posterior } \\
\text { border }\end{array}$ \\
\hline 1 & 2 & 6 & & & \\
\hline 2 & 2 & 1 & & 1 & 1 \\
\hline 3 & & 1 & & 7 & \\
\hline 4 & & 2 & 3 & 19 & 7 \\
\hline Total & 4 & 10 & 3 & 27 & 8 \\
\hline
\end{tabular}

Table 5. Results: correlation between severity and location of changes on longitudinal plains in patients with bilateral TSI

\begin{tabular}{|l|c|c|}
\hline Patient number & Right tibia & Left tibia \\
\hline 1 & 0 & $4 \mathrm{P}$ \\
\hline 2 & $1 \mathrm{M}$ & $1 \mathrm{M}$ \\
\hline 3 & $4 \mathrm{D}$ & $4 \mathrm{P}$ \\
\hline 4 & $3 \mathrm{D}$ & $4 \mathrm{M}$ \\
\hline 5 & $3 \mathrm{D}$ & $3 \mathrm{D}$ \\
\hline 6 & $1 \mathrm{D}, 4 \mathrm{P}$ & $4 \mathrm{P}$ \\
\hline 7 & 0 & $2 \mathrm{M}$ \\
\hline 8 & $1 \mathrm{D}$ & $1 \mathrm{D}$ \\
\hline 9 & $4 \mathrm{D}$ & $3 \mathrm{M}$ \\
\hline
\end{tabular}

Kijowski et al. [4] analysed the material of 142 tibias with TSI using the Fredericson classification system. He confirmed the bone marrow oedema located in medial cortex in $60 \%$ of cases of type $4 \mathrm{a}$ (with multifocal cortex abnormality localised in anterior and posterior cortex) and in the predominance of the cases in the types 1 to 3 (91\% in type $1,52 \%$ in type 2 , and $71 \%$ in type 3 ). The anterior cortex was involved in $6 \%$ of type $1,6 \%$ of type 3 , and $30 \%$ of type $4 \mathrm{a}$. In $75 \%$ of type $4 \mathrm{~b}$ cases the bone marrow oedema were located in the posterior cortex with the majority (28 of 38 cases) of liner areas (fracture line) in the posterior tibial cortex.

Most of our cases of TSI (38) were located on posterior and medial borders, and they were accompanied by oedematous changes on the same level. These cases were classified as more severe types (grade 3 and 4 ). The cases in type 4, with the majority located in posterior and medial border (29 in a group of 52 cases), were the same as the results obtained by Kijowski et al. classified as type $4 \mathrm{~b}$. Usually oedematous changes were seen at the same location as the cortical changes (on a longer span). Grade 4 changes may also be accompanied by oedematous changes along anterior and medial border (four of our cases) they may be newer than fractures or coexist with them, due to stress of lower intensity.

Early changes in grade 1 and 2 were noticed on anterior and medial sides of the tibia - in our material in 11 of the examined cases. More severe cases of grade 3 and 4 in this location were less frequent but were also accompanied by bone marrow oedema in the same location.

For type 3, seven of eight cases were located in the medial and posterior border; a similar location in comparison to type $4 \mathrm{a}$ described in the cited article. The rest of type 1 and 2 had similar location in the medial and anterior sides of the tibia.

We also did not notice changes on lateral borders of tibias on axial planes.

In running and in most other sports, both legs are usually under abnormal stress, so initially only one tibia may be affected, but later we can see changes in both legs. It should be considered to scan both legs in runners and military recruits even if the symptoms are unilateral or very subtle in the other leg because of the high probability of concomitant injury. In our group of 44 patients 35 patients underwent unilateral MRI exams, 9 patients bilateral. In bilateral exams only two tibiae showed no abnormality. In most exams the changes were bilateral, but in different locations and stages. In one tibia there were two locations of TSI in different stages; this was probably caused by long training with coexisting new and old changes. Hadid et al. [6] noticed bilateral changes in 12 of 26 patients, and Gaeta et al. [1] eight in 42 patients. We propose bilateral examination in young sportsmen even in unilateral signs and complaints, just to exclude bilateral changes.

There are many factors that may influence the incidence of stress injuries, such as weight, height, anatomy variants and disorders of the musculoskeletal system, defective posture, and training errors (bad technique of running, inappropriate shoes and surface, inadequate rest, etc.). Many theories try to describe the causes of the TSI and explain their location. In runners the most important muscles involved in applying forces during sport activity are the tibialis posterior, flexor digitorum longus, and soleus [7]. The most common locations of stress fracture in runners are medial and posterior borders of the tibia, where the compression action of muscles accumulates. A different type of stress occurs in jumpers and leapers, causing stress fracture of the anterior cortex [3]. The signs of periostitis on the anteromedial surface of the tibia are explained as the result of microtrauma of the cortical bone. The exact mechanism of acting forces during sport activity and their influence on soft-tissue and bone changes needs further investigation.

\section{Conclusions}

TSI may be suspected in young, healthy runners with lower leg pain on exertion. MRI techniques are the only method of identification of early symptoms of stress injuries. Such findings must be compared with signs and symptoms present during clinical examination, medical history of the patient, laboratory findings, and other exams. Knowledge of typical symptoms and locations of TSI 
are crucial in diagnosing and may prevent stress fracture. Analysing the results of our study we came to the conclusion that in the majority of cases of TSI in early stages anterior and medial borders of the tibia are affected, but later remodelling of the bone and stress fractures are mostly seen on posterior and medial borders.

In our group most TSI were seen in proximal and distal parts of tibiae. The differences in location of TSI on longitudinal and axial plains may be caused by the differ- entiated demographics of our group of patients. We propose a new, simplified classification of TSI and a protocol of scanning two tibiae even in a patient with unilateral exertional pain.

\section{Conflict of interest}

The authors report no conflict of interest.

\section{References}

1. Gaeta M, Minutoli F, Scribano E, et al. CT and MR imaging findings in athletes with early tibial stress injuries: Comparison with bone scintigraphy findings and emphasis on cortical abnormalities. Radiology 2005; 235: 553-561.

2. Taunton JE, Ryan MB, Clement DB, et al. A retrospective case-control analysis of 2002 running injuries. Br J Sports Med 2002; 36: 95-101.

3. Fredericson M, Bergman AG, Hoffman KL, et al. Tibial stress reaction in runners: correlation of clinical symptoms and scintigraphy with a new magnetic resonance imaging grading system. Am J Sports Med 1995; 23: 427-481.

4. Kijowski R, Choi J, Shinki K, et al. Validation of MRI classification system for tibial stress injuries. AJR Am J Roentgenol 2012; 198: 878-884.

5. Beck BR, Bergman AG, Arendt EA, et al. Tibial stress injury: relationship of radiographics, nuclear medicine bone scanning, MR Imaging, and CT severity grades to clinical severity and time to healing. Radiology 2012; 263: 811-818.

6. Hadid A, Moran DS, Evans RK, et al. Tibial stress changes in new combat recruits for special forces: patterns and timing at MR imaging. Radiology 2014; 273: 483-490.

7. Franklyn M, Oakes B. Aetiology and mechanisms of injury in medial tibial stress syndrome: Current and future developments. World J Orthop 2015; 6: 577-589. 